\title{
Die Frage nach dem Handeln Gottes in der Welt als elementares Glaubensproblem
}

\section{The Question of God's Action in the World as one Fundamental Aspect of Crisis of Faith}

\author{
CHRISTOPH BÖTTIGHEIMER \\ Theologische Fakultät \\ Katholische Universität Eichstätt-Ingolstadt \\ christoph.boettigheimer@ku.de
}

Zusammenfassung. Die christliche Religion beruht auf dem Handeln Gottes in der Welt, so wie es in der Heiligen Schrift vielfältig bezeugt wird. Doch kann heute in einer durchtechnisierten und durchrationalisierten Welt theologisch verantwortet vom Handeln Gottes gesprochen werden? Für nicht wenige Menschen sind die modernen naturwissenschaftlichen Erkenntnisse und technischen Errungenschaften Grund genug, nicht mehr mit einem Eingreifen Gottes in die Geschichte zu rechnen. Wie angesichts neuerer evolutionsbiologischer und kosmologischer Erkenntnisse dennoch an das Handeln Gottes in der Welt verantwortet geglaubt werden kann, ist die dem Artikel zugrunde liegende Fragestellung.

Stichworte: Offenbarung; Schöpfung; Anthropologie.

\begin{abstract}
Christian religion is mainly based on the fact of God's action in the world, as the Holy Bible expresses and testifies in many different ways throughout the salvation history. But how can we talk about God's action in the world in a responsible and reasonable way, while we found ourselves in a highly developed environment, were both, technical progress and rationality, determine our daily life? Evidently, more than just a few people consider science and technical progress to be the main reasons why the
\end{abstract}


belief in God's direct intervention in our world is not expected to happen any more. Therefore, the aim of this article is to point out how God's action in the world can be made plausible in a reasonable way with regard to recent research results in natural sciences, such as evolutionary biology or cosmology.

Keywords: revelation; creation; anthropology.

In der Heiligen Schrift, besonders im Alten Testament, fällt auf, dass sich Gott seinem Volk Israel durch geschichtliche Ereignisse, Taten und Widerfahrnisse offenbart. Er tat „große Wunder“, schlug „die Erstgeburt der Ägypter“, führte „Israel heraus[...] aus ihrer Mitte“, zerschnitt „das Schilfmeer [...] in zwei Teile“, stürzte „den Pharao ins Meer [...] samt seinem Heer“, schlug „große Könige“ und tötete „mächtige Könige“ (Ps 136). Der Gott der Väter ist also ein in der Geschichte Israels wirkender und sein Volk begleitender Gott. Im geschichtlichen Handeln gibt er sich kund und im Erretten und Befreien offenbart er seinen erlösenden Heilswillen - vor allem im Exodusgeschehen. Nach Ex 3,7-9 hat JHWH die Not des Volkes gesehen und den Klageruf gehört. Er ist demnach ein Gott, der sich der Menschen annimmt und sich um sie kümmert, vor allem dann, wenn sie in Bedrängnis sind.

Von den universalen Naturgesetzen und Wirkzusammenhängen konnte die Bibel natürlich noch keinerlei Kenntnis haben. Der heutige Mensch jedoch kann die biblische Geschichte unmöglich unter Absehung seiner naturwissenschaftlichen Erkenntnisse lesen und interpretieren; er kann nicht einfach wieder in die naturwissenschaftlich undifferenzierte Welt der Bibel eintauchen. Denn was früher Gott zugeschrieben wurde, kann oftmals heute auf rationale Weise erklärt werden. Die Welt wird als ein in sich geschlossenes System wahrgenommen, das ohne die Hypothese Gott auskommt, weshalb die Gottesfrage vielfach keinen Ansatz mehr im Denken des Menschen findet, und selbst Gläubige ihre Alltagsprobleme vor allem mit Hilfe von Wissenschaft und Technik zu lösen versuchen. Kann heute noch an einen Gott geglaubt werden, der „alles so herrlich regieret, der Dich auf Adelers Fittichen sicher geführet“, wie es in einem bekannten 
und viel gesungenen geistlichen Lied mit einem Text von Joachim Neander aus dem Jahr 1680 heißt?

Wird die physikalisch-materielle Wirklichkeit als ein eigengesetzliches, in sich geschlossenes Kausalsystem wahrgenommen und bei der Bewältigung der Alltagsaufgaben und lebenspraktischen Entscheidungen vorwiegend auf die wirtschaftliche, technische etc. Machbarkeit gesetzt, dann muss der Gottesglaube zwangsläufig bedeutungslos werden. Gott wird innerweltlich funktionslos, denn für sein besonderes Handeln bleibt kein Platz mehr. Wird aber dennoch am Gottesglauben festgehalten, weist dieser notgedrungen deistische Züge auf. Das bedeutet, Gottes Schöpfungstat wird auf den Beginn der Schöpfung eingegrenzt. Gott und Welt werden getrennt. Als der Deus otiosus überlässt der Schöpfergott die Welt ihren eigenen natürlichen Gesetzen, Funktionen und Mechanismen, mit der Konsequenz, dass es kein innerweltliches Wirken Gottes mehr geben kann, sondern nur noch den Lauf der Dinge. Tatsächlich meinen Soziologen seit einigen Jahren eine verstärkte Tendenz zum Deismus beobachten zu können. Göttliches und Weltliches werden getrennt und unterschiedlichen Bereichen zugeordnet, zwischen denen es keine Vermittlung gibt. Ein solches Verhalten impliziert allerdings einen latenten Hang zum praktischen Atheismus, denn hier kann mit einem Handeln Gottes in der Welt nicht mehr gerechnet werden. So ist der Begriff von Gott zwar noch vorhanden, doch spielt er in der Alltagswelt keine Rolle mehr, und Glaubensaussagen werden durch die Wirklichkeitserfahrungen nicht mehr eingeholt. Diese eher erfahrungsbezogene Problemanalyse soll nachfolgend theoriegestützt begründet werden.

\section{Kompetenzverlust des Gottesglaubens}

Nach dem französischen Philosophen und Soziologen Auguste Comte (1798-1857) ist die Reifung des menschlichen Bewusstseins einer gesetzmäßigen, evolutiven Entwicklung unterworfen, innerhalb der sich drei verschiedene Stadien unterscheiden lassen (Drei-Stadien-Gesetz).

Im theologischen oder fiktiven Stadium würde aufgrund einer natürlichen Neugierde nach der inneren Natur der Dinge gefragt. Dabei würden 
natürliche Erscheinungen auf übernatürliche Wesenheiten zurückgeführt. Das zweite Stadium sei das metaphysische oder abstrakte. Hier würden natürliche Phänomene auf Abstraktes zurückgeführt wie beispielsweise die Natur, die allgemeinen Ideen bei Platon oder das Wesen bei Aristoteles.

Im dritten, gegenwärtigen Stadium zähle allein das positive Denken, das menschliche Probleme lösen soll. Indem Phänomene kritisch beobachtet werden, könnten Ereignisse rational vorausgesehen werden. Weltbewältigung mittels Wissen („Know-how“) werde angestrebt. Diese Phase sei atheistisch, denn nun erzeuge der Mensch Herrschaftswissen und übe es aus, ohne dafür einen Gott zu brauchen. Die Aufgabe der kognitiven Leistung übernehme nun allein die exakte positive Wissenschaft. Dem Gottesglauben komme keine kognitive Kompetenz mehr zu; er habe keine Effektivität, keine eigenen Erkenntnismittel, keine eigene Wahrheit und keine eigene Funktion mehr. Durch das Fortschreiten des exakten Denkens würde jetzt auch der Mensch selbst immer mehr durchschaubar und damit auch die Theologie nach und nach überflüssig werden. Die Gottesfrage würde obsolet und aufhören als Frage weiter zu existieren.

Was Comte in weiser Voraussicht beschrieb, wird heute von Hans-Dieter Mutschler als Fakt konstatiert: „Die zunehmende Macht durch technische Weltbewältigung klappe die vertikale Dimension der traditionellen Religion in die horizontale des technischen Fortschritts, so dass nun plötzlich dieser Fortschritt sich mit religiösen Hoffnungen amalgamiere." (Mutschler (2009), 496). Die eigentliche Arbeit wird also von Menschen mittels Wissenschaft und Technik getan. An die Stelle Gottes tritt der Mensch, an die Stelle der Götter die Technik. Bis heute finden sich Erbteile dieses Ansatzes in unserem Weltverständnis wieder. Denn im alltäglichen Leben herrscht weithin die Grundüberzeugung vor - von esoterischen etc. Trends abgesehen -, dass die individuellen und beruflichen Herausforderungen wie auch gesellschaftliche Probleme mit Hilfe von Wissen(schaft) und Technik zu lösen sind. Man folgt dem Prinzip der geschlossenen Naturkausalität, wonach es keine Ursachen außerhalb dieses Systems geben kann. Wie kann in einer solchen technokratischen Welt noch angemessen von einem Handeln Gottes gesprochen werden? 


\section{Unvermitteltes Handeln Gottes?}

Angesichts des aufgezeigten Problemhorizonts steht die Frage im Raum, $o b$ und gegebenenfalls wie ein direktes Wirken Gottes in der Natur wissenschaftsverträglich gedacht werden kann. Glaubensaussagen zur unmittelbaren Wirkmächtigkeit Gottes machen ja nur Sinn, wenn sie mit der naturwissenschaftlichen Welterklärung als kompatibel aufgewiesen werden können. Bietet hierzu die von den Naturwissenschaften erkannte Nicht-Prognostizierbarkeit mancher Naturabläufe eine Möglichkeit?

Die naturwissenschaftliche Unberechenbarkeit bestimmter Naturprozesse im Mikro- und Makrobereich kann entweder mit Hilfe des der Natur inhärenten Zufalls geschehen und damit unter Aufgabe der linearen Determiniertheit der Natur, oder mit Hilfe des Hinweises auf die Unzulänglichkeit menschlicher Erkenntnisfähigkeit, verbunden mit dem Insistieren auf eine strikte Determiniertheit der Natur. Grundsätzlich kann die Frage, wie manche naturwissenschaftliche Nicht-Prognostizierbarkeit zu deuten sei - ontologisch / indeterministisch oder epistemologisch / deterministisch -, rein naturwissenschaftlich nicht entschieden werden. Die Antwort stellt bereits eine metaphysische Deutung physikalischer Prozesse dar. Aus diesem Grunde können beide Interpretationen naturwissenschaftlich vertreten werden.

Wird die Nicht-Prognostizierbarkeit bestimmter physikalischer Prozesse als kausale Erklärungslücke der Wirklichkeit gedeutet und theologisch mit Gottes freiem Handeln in Verbindung gebracht, macht sich der Gottesglaube eine Offenheit zu nutze, die als der Natur inhärent gedeutet wird. Ein direkter Konflikt mit naturwissenschaftlichen Erkenntnissen wäre damit ausgeschlossen. Würden also physikalische Unberechenbarkeiten, wie sie im quantenphysikalischen Mikrokosmos vorkommen, ontologisch gedeutet, sei, so u.a. der Quantentheoretiker Pascual Jordan (1902-1980) und der Physiker Karl Rawer (*1913), ein unvermitteltes Handeln Gottes prinzipiell nicht auszuschließen. Der Glaube an ein besonderes göttliches Handeln in der Welt könnte sich mit gesicherten naturwissenschaftlichen Erkenntnissen und den Naturgesetzmäßigkeiten vereinbaren lassen und 
wäre damit nicht irrational. Verortet man nämlich Gottes direktes Handeln in physikalischen Unbestimmtheiten, würden die physikalischen Erhaltungssätze und mithin das Wechselwirkungsprinzip nicht verletzt. Gott nimmt keine Lückenbüßer-Rolle ein, da er ja nicht in epistemischen Lükken, d.h. in dem Noch-nicht-Gewussten verortet wird, sondern in ontologischen Freiräumen. Quantenfluktuationen werden schließlich nicht als naturwissenschaftliche Erklärunglücken gedeutet, sondern im Sinne einer unaufhebbaren Offenheit und Flexibilität der quantenphysikalischen Welt.

Die Frage allerdings, wie eine nicht-materielle Entität auf Materielles einwirken kann, bleibt bislang unbeantwortet. Des Weiteren bleibt ungeklärt, wie man sich ein solches Wirken Gottes näher vorzustellen hat. Wie kann Gottes direktes Handeln in der Welt einerseits auf quantenphysikalische Indeterminismusphänomene eingegrenzt werden, andererseits aber zugleich eine Relevanz für das gesamte, sich evolutiv entwickelnde Universum behauptet werden? Außerdem gibt es keine ausgearbeitete Theorie, die die Konsequenzen mikrophysikalischer Ereignisse im makrophysikalischen Bereich erklären könnte. Nicht zuletzt drängt sich hier der Verdacht auf, dass Gott letztlich zu einer geschöpflichen Ursache oder kontingenten Naturkraft wird. Wenn das Handeln Gottes in Entsprechung zu natürlichen Ursachen gedacht und auf (quanten-)physikalische Ereignisse bezogen wird, wird seine Transzendenz verkannt: Gott ist die Bedingung der Möglichkeit aller (quanten-)physikalischen Ereignisse und keine in der Natur wirkende causa analog zu anderen natürlichen causae.

Darüber hinaus ruft die Annahme eines besonderen und unvermittelten Handelns Gottes immer die schwerwiegende Frage hervor, weshalb Gott, wenn er denn die Möglichkeit des direkten Interagierens im Rahmen der Nicht-Prognostizierbarkeit bestimmter physikalischer Prozesse besitzt, nur da und dort eingreift. Ist sein Handeln von Willkür geprägt? Der Gedanke der Willkürlichkeit würde sich auch insofern nahelegen, als der Zufall ja ein intentionales Handeln grundsätzlich ausschließt. Außerdem scheint es widersprüchlich, irreduzierbare Wahrscheinlichkeit in bestimmten quantenphysikalischen Prozessen mit einem allwissenden Gott in Verbindung bringen zu wollen. All diese Anfragen verdeutlichen, dass bislang 
kein theologisches Konzept vorliegt, das die Rede von einem punktuellen, innovatorischen Handeln eines transzendenten Gottes mit Hilfe quantentheoretischer Erkenntnisse überzeugend zur Darstellung bringt.

Die naturwissenschaftliche Erkenntnis besagt, dass die Natur Indeterminismen aufweist und dass die Naturwissenschaft keineswegs die Wirklichkeit im Ganzen im Blick hat. So kann seitens der Naturwissenschaft ein besonderes und unvermitteltes Handeln Gottes nicht generell ausgeschlossen werden, wenngleich ein solches innovatorisches, exklusives Handeln naturwissenschaftlich gegenwärtig kaum plausibel gemacht werden kann. Solange aber die Theologie keine mit naturwissenschaftlichen Ergebnissen verträgliche und theologisch akzeptable Erklärung für ein innovatorisches Handeln Gottes bereithalten kann, scheint es zumindest schwierig, auf verantwortete Weise von einem unmittelbaren Handeln Gottes in der Welt auszugehen, das über ein schöpferisches und personal vermitteltes Wirken hinausreicht. Dennoch aber kann es nicht prinzipiell ausgeschlossen werden, sofern Gott als der semper maior ernstgenommen und alle theologische Erkenntnis letztlich als docta ignorantia begriffen wird.

\section{Schöpferisches Handeln Gottes}

Unabhängig von der Nicht-Prognostizierbarkeit bestimmter physikalischer Prozesse ist Gottes Handeln zunächst darauf zu beziehen, dass er kraft seines Wortes (Röm 4,17) alles ins Dasein gerufen hat und die gesamte Schöpfung durchwirkt, ohne sie ihrer Eigenständigkeit und Eigengesetzlichkeit zu berauben. Gott ist Konstitutionsgrund der Welt, ohne das Geschehen in ihr direkt und unmittelbar zu verursachen. Der Schöpfungsglaube besagt, dass sich alles Sein Gott verdankt und dauernd, kontinuierlich von ihm aus freier Liebe im Sein erhalten wird, was den Rückfall ins Nichts verhindert inklusive aller Evolutionsprozesse. Gottes schöpferisches Tätigsein ist die Bedingung der Möglichkeit aller evolutiven Entwicklungen.

Ausgangspunkt des Schöpfungsbegriffs ist die objektive Kontingenz der Welt und sein Inhalt die denknotwendige ontologische Erklärung hierfür. Das bedeutet, dass sich das Wort Kausalität zur Bezeichnung dessen, 
was mit Schöpfung gemeint ist, wenig eignet. Der Schöpfungsbegriff hat nichts mit dem Schema ,Ursache-Wirkung' zu tun, weshalb eine Verknüpfung von Urknalltheorie und Schöpfungslehre grundlegend falsch wäre. Die biblischen Schöpfungserzählungen klären nicht die Frage nach einer urzeitlichen Verursachung, die dann womöglich im Urknall festzumachen und zeitlich zu umschreiben wäre; stattdessen beschreiben sie ein letztes und tiefstes Getragensein der Welt durch den freien, Ursprung setzenden Schöpfungsakt Gottes. Damit machen sie eine grundsätzliche Sinnaussage in Bezug auf die Wirklichkeit. Insofern sie allein auf Gott als dem absoluten Seinsgrund beruht, kann sie nur als gut und sinnvoll gedacht werden.

Die Schöpfungsaussage kommt ohne jede zeitliche Angabe aus. Schon Augustinus bemerkte, dass Gott die Welt nicht in tempore geschaffen habe, sondern cum tempore. Die Zeit ist mit der Welt geschaffen, so dass die Frage nach dem der Wirklichkeit vorausgehenden Anfang obsolet wird. Auf die Frage: „,Was tat Gott, bevor er Himmel und Erde erschuf?““ antwortet Augustinus darum zunächst scherzhaft: „,Er hat Höllen hergerichtet für Leute, die so hohe Geheimnisse ergrübeln wollen'“ (Conf. XI, 12,14), und er fügt später hinzu, dass „vor Himmel und Erde Zeit überhaupt nicht war, was soll dann die Frage, was Du ,damals' tatest? Es gab kein ,Damals‘, wo es Zeit nicht gab.“ (Conf. XI, 13,15) Vor der Materie gab es also keine Zeit. Selbst unter der Annahme, die Welt sei zeitlich ohne Anfang bzw. die Materie ewig, wird die metaphysische Wahrheit der Schöpfungsaussage, die ja die andauernde Möglichkeitsbedingung aller Wirklichkeit benennt, nicht in Frage gestellt. Mit anderen Worten: Das Schöpfungshandeln Gottes, bei dem zusammen mit den geschaffenen Dingen erst die Zeit entsteht, ist selbst durch keine Zeit bestimmt, es muss mithin ewig sein. So bemerkte wiederum Augustinus: „Du aber, Herr, wirkest immer und ruhest immer, Du siehst nicht in der Zeit noch regst Du Dich zuzeiten und ruhst zuzeiten. Und doch bist Du es, der da wirkt den Unbestand des Sehens in der Zeit und die Zeiten selber und das Ruhen, wann Zeit nicht mehr sein wird." (Conf. XIII 37,52) Wie also das schöpferische Handeln Gottes nicht in der Zeit zu denken ist, da die Zeit erst mit der Schöpfung kommt, darf auch Gott nicht zeitlich vor der Schöpfung gedacht werden, 
d.h. es gibt keine Zeit, in der Gott noch nicht Schöpfer war, vielmehr ist er der ewige Schöpfergott.

Das zeitlose schöpferische Handeln Gottes liegt aller Wirklichkeit zugrunde, nicht voraus, weshalb es sich weder datieren noch historisieren und damit auch nicht einfach in naturwissenschaftliche Kategorien transformieren lässt. Das aber bedeutet, dass das schöpferische Handeln Gottes als die Bedingung der Möglichkeit aller kosmologischen und evolutiven Entwicklung als andauernd zu denken ist. Dabei ist der Zufall kein Gegenspieler Gottes, sondern vielmehr Ausdruck der gottgewollten Freiheit der Schöpfung. Schöpfungstheologisch kommt alles vom frei handelnden Gott her und findet in ihm sein Ziel, ohne dass im Kausalzusammenhang die Schöpfung zur göttlichen Marionette und damit Freiheit zur Illusion wird. Die Freiheit ernst zu nehmen bedeutet, der Materie letzten Endes selbst zuzutrauen, dass sie dazu fähig ist, sich zu überschreiten und sogar Geist hervorzubringen. „Gott zwingt die Dinge nicht in eine bestimmte Richtung, sondern lädt ein, wirbt, lockt: Alles in der Welt vom Urknall an geschieht in einer ständigen Interaktion zwischen Gott (als ermöglichendem Grund) und den (freigegebenen) Geschöpfen, in einem mehr oder weniger gut gelingenden und oft auch misslingenden ,Dialog'. Ein solch ,dialogisches Verhältnis Gott-Welt ist nicht erst auf der Ebene des Menschen anzunehmen, sondern - in analoger und graduell abgestufter Weise - schon im vormenschlichen Bereich und im kosmischen Prozess von Anfang an." (Kessler (32010), 134)

Es ist Gott, der die von ihm geschaffenen Dinge fortwährend im Dasein erhält und ihnen das Sein als etwas Eigenes schenkt, jedoch raubt er ihnen ihre Eigenständigkeit und Eigengesetzlichkeit nicht. Mit anderen Worten: Das kontinuierliche Schöpferwirken Gottes setzt die Eigendynamik und Autonomie der Welt nicht außer Kraft, sondern ermöglicht diese erst. Durch sein ständiges schöpferisches Wirken erhält Gott die Schöpfung im Dasein und ist so im gesamten raumzeitlichen Prozess des evolvierenden Kosmos gegenwärtig und präsent. Als Schöpfer ist Gott seiner Schöpfung bleibend zugewandt, in ihr ist er allgegenwärtig, ohne empirisch wissenschaftlich objektiviert oder gar mit einzelnen Naturprozessen oder Ge- 
schichtsabläufen in einen unmittelbaren Zusammenhang gebracht werden zu können.

In der Schöpfung drückt sich Gott selbst aus, durch sie spricht er den Menschen an. Ist Gott eine „Hintergrundstimme“, die die sprachlich verfasste Wirklichkeit trägt, auf die Evolution des Bewusstseins, die Geistregung und die Kultur einwirkt und sich geschichtlich mittels des Menschenwortes zu Wort meldet? Wenn dem so ist, dann müsste versucht werden, im Gewöhnlichen das Wunderbare zu entdecken. Das Leben sollte in der Gegenwart Gottes bewusst gelebt und alles in der mystischen Grundhaltung getan werden, dass der Mensch sein Dasein Gott verdankt und von ihm bleibend abhängig ist. Jede Handlung müsste im Sinne der Selbsthingabe an Gott vollbracht und so zum Gebet werden, gerade wie Paulus uns auffordert, „ohne Unterlaß“ zu beten (1 Thess 5,17). „Alles, was ihr tut in Wort oder Werk, tut alles im Namen des Herrn Jesus und dankt durch ihn Gott, dem Vater." (Kol 3,17) Einer Spiritualität die damit ernst macht, dass in allem Bedingten das Unbedingte mit erfahren wird, steht allerdings nicht selten die modernen Lebensauffassung entgegen, gemäß der die Gottesfrage - wenn überhaupt - erst gestellt wird, wenn sich die berechenbaren und als selbstverständlich erachteten Naturabläufe menschlicher Kontrolle entziehen.

\section{Personal vermitteltes Wirken Gottes}

Ist Gott nur kraft seines Schöpfungsaktes in der Welt anwesend? Nein, seine unbesiegbare Liebe kann auch personal vermittelt am Werk gedacht werden. Nach biblischem Zeugnis handelt Gott frei in der Geschichte, indem er sich bestimmten Menschen mitteilt, sie anspricht und beruft: Mose, Propheten, Paulus oder die Apostel, von denen es im sekundären Markusschluss heißt: „Sie aber zogen aus und predigten überall. Der Herr stand ihnen bei und bekräftigte die Verkündigung durch die Zeichen, die er geschehen ließ.“ (Mk 16,20)

Ein personal vermitteltes Handeln Gottes ist dort anzunehmen, wo sich Menschen in ihrem Tun auf Gott hin öffnen. Statt in sich selbst verhaftet 
und auf ihre begrenzten Möglichkeiten beschränkt zu bleiben, wissen sie sich von Gottes Geist und Liebe ergriffen und stellen sich ganz bewusst in den Dienst des göttlichen Willens. Wie die Liebe den Menschen inspirieren und motivieren kann, so kann auch das Handeln Gottes in der Geschichte gedacht werden. Immer wieder erfahren Menschen, dass sie von fremden, überpersonalen Mächten und Gewalten heimgesucht und ergriffen werden, sei es negativ von der Macht des Bösen oder positiv von der Macht der Liebe. Gott handelt durch Menschen, die sich von Gottes Geist ergreifen lassen und seinen Willen tun. Das durch Menschen vermittelte Geistwirken Gottes ist ein geschichtswirksames Handeln; es verändert nicht nur den einzelnen Menschen, sondern durch ihn auch das gesamte Weltgefüge. $\mathrm{Zu}$ -

gleich wird durch ein solches personal vermitteltes Handeln Gottes weder unsere Freiheit negiert, noch werden naturwissenschaftliche Erkenntnisse in Frage gestellt.

Im menschlichen Handeln kann sich die göttliche Wirklichkeit manifestieren. Es ist Gottes eigenes Wesen, seine Liebe, die Menschen zum Handeln bewegt, ohne sie zu zwingen - eine Liebe, die den Kern des geschichtlichen Heilswirkens Gottes bildet und in Jesus von Nazareth Fleisch angenommen hat. Fortan wirkt diese Liebe kraft des Geistes Gottes innerweltlich fort und kann freiwillig angenommen werden. Inmitten der Welt kann also Gott am Werk erfahren werden, wo immer seinem Geist Raum gegeben wird, vermittelt durch einen selbst oder durch den Anderen. Wichtig ist die Feststellung, dass das Wirken Gottes in Welt und Geschichte hier nicht durch die Suche nach Lücken in der Evolution geschieht, sondern in Kompatibilität zur Naturwissenschaft. Die Handlungskausalität, die für uns Menschen unverzichtbar ist, bleibt ja für die Naturwissenschaft unerreichbar. Außen vor bleiben Absicht und Sinn, Motive und Ziele menschlicher Handlungen.

\section{Ausblick}

Die Theologie kann heute nicht mehr davon ausgehen, dass die Möglichkeit einer zeitweisen Außerkraftsetzung der Naturgesetzmäßigkeit durch 
Gott problemlos denkbar wäre. Auch wenn ein streng deterministisches Naturverständnis als überwunden gelten kann, so hilft dieses Wissen nur bedingt, um die Rede eines besonderen, interventionistischen Handelns Gottes in der Welt zu explizieren. Denn kann in bestimmten Fällen ein Ursache-Wirkzusammenhang auch nicht unmittelbar ausgemacht werden, so gilt doch als unbezweifelbare naturwissenschaftliche Erkenntnis, dass die physikalische Welt in sich kausale geschlossen ist. Demnach können die Ursachen für physikalische Prozesse nur in der Welt selbst liegen.

Nach dem Zeugnis der Hl. Schrift ist die Welt Gottes Schöpfung, in der er schöpferisch gegenwärtig, mehr aber noch personal vermittelnd am Werk ist. Auf das in der Geschichte sich vollziehende Handeln Gottes lenken die biblischen Glaubensaussagen unser Augenmerk, vollziehen sich darin doch die Erlösung und das Heil der Menschen. Dieses Handeln Gottes gegenüber der Vernunft unter Bezugnahme auf naturwissenschaftliche Erkenntnisse plausibel zu machen, ist Aufgabe der Fundamentaltheologie. Indem sie dabei die Schwierigkeiten mit dem Gedanken eines unmittelbaren Handelns Gottes in der Welt offen benennt, kann sie einen wertvollen Beitrag zur Lösung jener Glaubensprobleme leisten, die daraus resultieren, dass die Welt des Glaubens und die des Wissens heute teils nur noch schwer miteinander vermittelt werden können.

\section{References}

Becker, Patrick. 2009. “Ein wirkmächtiger Gott? Ein Problemaufriss aus theologischer Perspektive." In Gottesbilder an der Grenze zwischen Naturwissenschaft und Theologie, edited by G. Souvignier u.a., 83-93. Darmstadt: Wissenschaftliche Buchgesellschaft.

Becker, Patrick. 2005. "Das Wirken Gottes in der Welt. Zur Vereinbarkeit von Theismus und Naturwissenschaften." Theologische Zeitschrift 56: 248-257.

Bernhardt, Reinhold. 2008. "Durchbrochene Naturgesetze? Heute an das Handeln Gottes glauben." In Herder Korrespondenz Spezial - Getrennte Welten? Der Glaube und die Naturwissenschaften, 18-22. Freiburg i. Br.: Herder.

Böttigheimer, Christoph. 2013. Wie handelt Gott in der Welt? Reflexionen im Spannungsfeld von Theologie und. Freiburg i. Br.: Herder. 
Hünermann, Peter. 2013. “Gottes Handeln in der Geschichte. Theologie als Interpretatio temporis." In Phänomenologie und Theologie im Gespräch. Impulse von Bernhard Welte und Klaus Hemmerle, edited by G. Bausenhart, M. Böhnke, D. Lorenz, 560-593. Freiburg i. Br.: Herder.

Kessler, Hans. 2006. Den verborgenen Gott suchen. Gottesglaube in einer von Naturwissenschaften und Religionskritik geprägten Welt. Paderborn: Schöningh $\mathrm{Pa}-$ derborn.

Kessler, Hans. 2010. Evolution und Schöpfung in neuer Sicht. Kevelaer: Butzon \& Bercker.

Loichinger, Alexander. 2003. "Wirken Gottes und moderne Naturwissenschaften." In Theologie und Glaube 46: 82-95.

Lüke, Ulrich. 2004. "Schöpfung aus dem Nichts oder fortlaufende Schöpfung?.“ In Kosmologie - Fragen nach Evolution und Eschatologie der Welt, edited by H. A. Müller, 39-52. Göttingen: Vandenhoeck \& Ruprecht.

Mutschler, Hans-Dieter. 2009. "Europa: Ökologie als Thema theologischer Reflexion.“ Herder Korrespondenz 63: 495-497.

Negel, Joachim, "Weil die Welt nicht ganz dicht ist ... Eine philosophisch-theologische Erörterung der Frage nach dem Wirken Gottes in der Welt." In Bittgebet (QD 256 ), edited by W. Eisele, 102-185. Freiburg i. Br.: Herder.

Peacocke, Arthur. 1998. Gottes Wirken in der Welt. Theologie im Zeitalter der Naturwissenschaft. Mainz: Matthias Grünewald.

Stosch, Klaus von. 2006. Gott - Macht - Geschichte. Versuch einer theodizeesensiblen Rede vom Handeln Gottes in der Welt. Freiburg i. Br.: Herder.

Weissmahr, Béla. 1997. “Gottes Wirken in der Welt - das Verhältnis von göttlicher und innerweltlicher Ursache." In Glaube im Kontext naturwissenschaftlicher Vernunft, edited by R. Isak, 23-42. Freiburg i. Br.: Herder. 